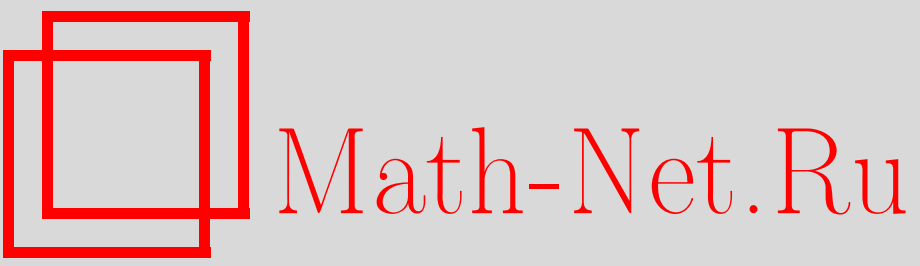

В. Г. Чирский, Алгебраические свойства точек некоторого бесконечномерного метрического пространства, Итоги науки и техн. Сер. Соврем. мат. и ее прил. Темат. обз., 2020, том 179, 81-87

DOI: https://doi.org/10.36535/0233-6723-2020-179-81-87

Использование Общероссийского математического портала Math-Net.Ru подразумевает, что вы прочитали и согласны с пользовательским соглашением

http://www.mathnet.ru/rus/agreement

Параметры загрузки:

IP: 3.91 .87 .62

26 апреля 2023 г., 16:28:02 


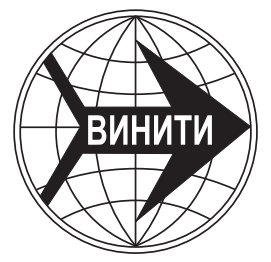

\title{
АЛГЕБРАИЧЕСКИЕ СВОЙСТВА ТОЧЕК НЕКОТОРОГО БЕСКОНЕЧНОМЕРНОГО МЕТРИЧЕСКОГО ПРОСТРАНСТВА
}

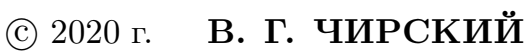

АннотАция. В статье сообщается о развитии теории трансцендентных чисел в полиадической области, представляющей собой бесконечномерное метрическое пространство - прямое произведение полей $p$-адических чисел.

Ключевые слова: бесконечная алгебраическая независимость, $F$-ряд.

\section{ALGEBRAIC PROPERTIES OF POINTS OF SOME INFINITE-DIMENSIONAL METRIC SPACE}

\author{
(c) 2020 V. G. CHIRSKII
}

\begin{abstract}
In this paper, we report on the development of the theory of transcendental numbers in a polyadic domain, which is an infinite-dimensional metric space, namely, the direct product of fields of p-adic numbers.
\end{abstract}

Keywords and phrases: infinite algebraic independence, $F$-series.

AMS Subject Classification: 11J91, 11J61

1. Множество полиадических чисел и его обобщения. Рассмотрим множество точек вида $\left(x_{1}, x_{2}, \ldots, x_{n}, \ldots\right)$, образующих бесконечномерное пространство. Это пространство представляет собой прямое произведение полей $p$-адических чисел. Именно, координата с номером $n$ этого вектора представляет собой $p_{n}$-адическое число, где $p_{n}$ - простое число с номером $n$. Напомним, что $p$-адические числа являются пополнением поля рациональных чисел по $p$-адическому нормированию. Для рационального числа $a$ символ $|a|_{p}$ обозначает величину $p$-адического нормирования, т.е. $|a|_{p}=p^{-\vartheta_{p}(a)}$, где, в свою очередь, символ $\vartheta_{p}(a)$ обозначает степень, в которой простое число $p$ входит в разложение числа $a$ на простые множители. Это прямое произведение имеет естественную структуру коммутативного кольца с единицей (и с делителями нуля). Его принято называть кольцом полиадических чисел (см. [5]).

Это кольцо допускает другие описания. Введем на кольце целых чисел $\mathbb{Z}$ наименьшую топологию, инвариантную относительно сдвига и такую, что идеалы $m \mathbb{Z}, m \in \mathbb{Z}$ кольца $\mathbb{Z}$ образуют базу системы окрестностей нуля. При этом операции сложения и умножения непрерывны, и кольцо целых чисел с введенной топологией имеет структуру топологического кольца. На этом топологическом кольце можно ввести метрику, полагая

$$
\rho(x, y)=\sum_{m=1}^{\infty} \frac{1}{2^{m}}\left\lfloor\frac{x-y}{m}\right\rfloor
$$

Работа выполнена при поддержке проекта Московского государственного университета им. М. В. Ломоносова «Современные проблемы фундаментальной математики и механики». 
где символ $\lfloor t\rfloor$ обозначает расстояние от числа $t$ до ближайшего к нему целого числа. Бесконечную последовательность целых чисел $\left\{a_{1}, a_{2}, \ldots, a_{n}, \ldots\right\}$ назовем фундаментальной, если для любого натурального числа $k$ существует натуральное число $N$ такое, что для любых натуральных $n$, $m$, больших $N, a_{n} \equiv a_{m}(\bmod k !)$. Полученное метрическое пространство не является полным. Например, последовательность $1 !, 1 !+2 !, 1 !+2 !+3 !, \ldots$ является фундаментальной, но не имеет предела в построенном топологическом кольце. Фундаментальные последовательности образуют кольцо. Понимая предел в смысле введенной выше топологии, назовем последовательность целых чисел $\left\{a_{1}, a_{2}, \ldots, a_{n}, \ldots\right\}$ нулевой последовательностью, если

$$
\lim _{n \rightarrow \infty} a_{n}=0 .
$$

Фундаментальные последовательности называются эквивалентными, если их разность - нулевая последовательность. Кольцо целых полиадических чисел определяется как кольцо эквивалентных фундаментальных последовательностей. Введенная выше метрика (1) топологического кольца целых чисел естественно продолжается на кольцо полиадических чисел.

Элементы a кольца целых полиадических чисел имеют каноническое представление в виде

$$
\mathfrak{a}=\sum_{m=1}^{\infty} a_{m} m !, \quad a_{m} \in \mathbb{N}, 0 \leqslant a_{m} \leqslant m .
$$

Степень, в которой простое число $p$ входит в разложение числа $n$ ! на простые множители, равна $\frac{n-S_{n}}{p-1}$, где $S_{n}$ обозначает сумму цифр в $p$-ичном разложении числа $n$. Следовательно, для любого $p$ при $n \rightarrow \infty$ выполняется соотношение $\left|a_{n} n !\right|_{p} \rightarrow 0$. Это - достаточное условие для того, чтобы ряд $(2)$ сходился в поле $\mathbb{Q}_{p}$. Сумму этого ряда $(2)$ в поле $\mathbb{Q}_{p}$ обозначаем $\mathfrak{a}^{(p)}$. Как отмечено выше, полиадическое число $\mathfrak{a}$ можно рассматривать как точку бесконечномерного пространства с координатами $\mathfrak{a}^{\left(p_{n}\right)}$, где $p_{n}$ - простое число с номером $n$.

Приведем краткий (и неполный) обзор задач, связанных с теорией полиадических чисел. Любое натуральное число $M$ допускает единственное представление в виде

$$
M=\sum_{m=1}^{N} a_{m} m !, \quad a_{m} \in \mathbb{N}, 0 \leqslant a_{m} \leqslant m .
$$

Это представление называют факториальным. Свойства факториального представления, в сравнении со свойствами так называемого представления с двойной базой, исследованы в [14]. Эти представления актуальны при решении некоторых практических задач.

С помощью меры Хаара-Лебега $\mu$ на кольце полиадических чисел можно определить интегрирование (см. например, [5]). В [4] доказано, что последовательность натуральных чисел равномерно распределена в кольце целых полиадических чисел. Это означает, что для любой непрерывной на этом кольце функции $f(x)$ выполняется равенство

$$
\lim _{N \rightarrow \infty} \frac{1}{N} \sum_{n=1}^{N} f(n)=\int f(x) d \mu .
$$

В книге [5] дан обзор ряда других важных результатов о свойствах кольца полиадических чисел и функций, определенных на этом кольце.

Отметим роль кольца полиадических чисел в ряде алгебраических задач (подробнее см. [9]).

Следует отметить, что ряды вида

$$
\sum_{n=0}^{\infty} a_{n} n !, \quad a_{n} \in \mathbb{Q},
$$

могут сходиться не во всех полях $\mathbb{Q}_{p}$. В случае, когда такой ряд расходится лишь в конечном множестве полей $\mathbb{Q}_{p}$, мы говорим о почти полиадических числах и отождествляем их с элементами прямого произведения всех полей $\mathbb{Q}_{p}$, кроме упомянутого выше конечного множества. 
Можно рассмотреть и прямое произведение полей $\mathbb{K}_{v}$, где $v$ продолжает $p$-адическое нормирование поля $\mathbb{Q}$ на поле $\mathbb{K}$ алгебраических чисел конечной степени $\varkappa$. Если $v$ продолжает $p$-адическое нормирование, то поле $\mathbb{K}_{v}$ представляет собой алгебраическое расширение поля $p$-адических чисел $\mathbb{Q}_{p}$ степени $\varkappa_{v}$ и справедливо равенство $\sum_{v} \varkappa_{v}=\varkappa$, где суммирование производится по всем нормированиям $v$, продолжающим $p$-адическое нормирование. Мы рассматриваем нормализованные нормирования, для которых $|p|_{v}=p^{-\varkappa v} / \varkappa$. Если нормирование $v$ продолжает обычную абсолютную величину, то ему соответствует алгебраически сопряженное поле $\mathbb{K}^{(i)}$ и $\varkappa_{v}=1$, если $\mathbb{K}^{(i)}$ - подполе поля действительных чисел, и $\varkappa_{v}=2$, если нет. Равенство $\sum_{v} \varkappa_{v}=\varkappa$ выполняется и в этом случае.

При этом мы будем говорить о $\mathbb{K}$-полиадических и соответственно о $\mathbb{K}$-почти полиадических числах.

2. Алгебраические свойства элементов бесконечных прямых произведений. Глобальные соотношения. Предположим, что $\alpha \in \mathbb{K}$ и что ряд

$$
\sum_{n=0}^{\infty} c_{n} \cdot n ! \cdot \alpha^{n}
$$

сходится в поле $\mathbb{K}_{v}$ для всех нормирований $v$ поля $\mathbb{K}$, продолжающих $p$-адическое нормирование, для всех простых чисел $p$, кроме, возможно, конечного их числа. Как отмечено выше, это позволяет рассматривать этот ряд как элемент бесконечного прямого произведения этих полей $\mathbb{K}_{v}$. Это прямое произведение имеет естественную структуру кольца, операциям в котором соответствуют операции по каждой координате. Для элемента $\mathfrak{a}$ этого прямого произведения обозначаем $\mathfrak{a}^{(v)}$ его координату в поле $\mathbb{K}_{v}$.

Если существует $P(x)$ - многочлен с рациональными коэффициентами, отличный от тождественного нуля такой, что $P(\mathfrak{a})=0$ (иными словами, $P\left(\mathfrak{a}^{(v)}\right)=0$ в каждом поле $\mathbb{K}_{v}$ этого прямого произведения), то говорим, что $\mathfrak{a}-$ алгебраический элемент. Если элемент $\mathfrak{a}$ не является алгебраическим, то его называют трансцендентным. Трансцендентность элемента означает, что для любого $P(x)$ - многочлена с рациональными коэффициентами, отличного от тождественного нуля, существует простое число $p$ и нормирование $v$ поля $\mathbb{K}$, продолжающее $p$-адическое нормирование поля $\mathbb{Q}$ такие, что $P\left(\mathfrak{a}^{(v)}\right) \neq 0$ в поле $\mathbb{K}_{v}$. Назовем элемент $\mathfrak{a}$ бесконечно трансцендентным, если для любого $P(x)$ - многочлена с рациональными коэффициентами, отличного от тождественного нуля, существует бесконечное множество простых чисел $p$, для каждого из которых есть нормирование $v$ поля $\mathbb{K}$, продолжающее $p$-адическое нормирование, такое, что $P\left(\mathfrak{a}^{(v)}\right) \neq 0$ в поле $\mathbb{K}_{v}$. Элемент a называется глобально трансцендентным, если для любого $P(x)$ - многочлена с рациональными коэффициентами, отличного от тождественного нуля, неравенство $P\left(\mathfrak{a}^{(v)}\right) \neq 0$ выполняется во всех полях $\mathbb{K}_{v}$ рассматриваемого прямого произведения.

Примерами глобально трансцендентных чисел служат ряды

$$
\sum_{k=0}^{\infty} n_{k} !
$$

где $\left\{n_{k}\right\}$ - достаточно быстро растущая последовательность натуральных чисел. Более того, ряды

$$
\sum_{k=0}^{\infty} \frac{n_{k} !}{p_{k}^{m_{k}}},
$$

в которых $p_{k}$-простое число с номером $k$, a $\left\{n_{k}\right\},\left\{m_{k}\right\}$ - достаточно быстро растущие последовательности натуральных чисел, дают примеры чисел лиувиллевых, следовательно, трансцендентных как в поле $\mathbb{R}$, так и в любом поле $\mathbb{Q}_{p}$. Действительно, быстрый рост последовательности $\left\{n_{k}\right\}$ обеспечивает трансцендентность суммы этого ряда в любом поле $\mathbb{Q}_{p}$, а быстрый рост последовательности $\left\{m_{k}\right\}$ - трансцендентность суммы этого ряда в поле $\mathbb{R}$.

Отметим, что из бесконечной трансцендентности элемента не следует трансцендентность $\mathfrak{a}^{(v)}$ хотя бы для одного простого числа $p$ и нормирования $v$ поля $\mathbb{K}$, продолжающего $p$-адическое 
нормирование поля $\mathbb{Q}$. Например, элемент $(1,2, \ldots, n, \ldots)$ прямого произведения полей $\mathbb{Q}_{p}$ (координата которого равна $n$ в поле $\mathbb{Q}_{p_{n}}$, соответствующем $n$-му простому числу $p_{n}$ ) бесконечно трансцендентен. Действительно, любой $P(x)$-многочлен с рациональными коэффициентами, отличный от тождественного нуля, может обратиться в ноль лишь на конечном множестве натуральных чисел.

Для совокупности элементов $\mathfrak{a}_{1}, \ldots, \mathfrak{a}_{m}$ аналогичным образом определяются понятия их алгебраической зависимости, алгебраической независимости, бесконечной алгебраической независимости и глобальной алгебраической независимости. Достаточно вместо многочлена $P(x)$ рассмотреть $P\left(x_{1}, \ldots, x_{m}\right)$ - многочлен с рациональными коэффициентами, отличный от тождественного нуля.

Ряд, члены которого - алгебраические числа, сходящийся в различных полях $\mathbb{K}_{v}$, имеет суммами элементы этих полей $\mathbb{K}_{v}$. При этом может оказаться, что в различных полях $\mathbb{K}_{v}$ его суммой являются различные элементы поля $\mathbb{K}$. Например, ряд $(1-(-15))^{1 / 2}$ сходится в поле $\mathbb{Q}_{3}$ к рациональному числу $1 / 2$, а в поле $\mathbb{Q}_{5}-$ к числу $-1 / 2$. Вместе с тем ряд, члены которого алгебраические числа, сходящийся в различных полях $\mathbb{K}_{v}$, может иметь в них суммой один и тот же элемент поля $\mathbb{K}$. Например, в любом поле $\mathbb{Q}_{p}$ выполняется равенство

$$
\sum_{n=1}^{\infty} n \cdot n !=-1
$$

Эта ситуация является частным случаем введенного в 1981 г. Э. Бомбьери в [18] понятия глобального (алгебраического) соотношения.

Пусть $P\left(y_{1}, \ldots, y_{m}\right)$ - отличный от нулевого многочлен с коэффициентами из поля $\mathbb{K}$, и пусть степенные ряды $f_{1}(z), \ldots, f_{m}(z)$ имеют коэффициенты из поля $\mathbb{K}$ и точка $\alpha \in \mathbb{K}$. Соотношение

$$
P\left(f_{1}(\alpha), \ldots, f_{m}(\alpha)\right)=0
$$

называется глобальным, если оно выполняется во всех полях $\mathbb{K}_{v}$, в которых сходятся все ряды $f_{1}(\alpha), \ldots, f_{m}(\alpha)$. Используя данные выше определения, получаем, что глобальное соотношение (3) означает алгебраическую зависимость над полем $\mathbb{K}$ элементов $f_{1}(\alpha), \ldots, f_{m}(\alpha)$ прямого произведения всех полей $\mathbb{K}_{v}$, в которых сходятся все ряды $f_{1}(\alpha), \ldots, f_{m}(\alpha)$.

Известное равенство

$$
\sum_{n=1}^{\infty} n \cdot n !=-1,
$$

верное во всех полях $\mathbb{Q}_{p}$, дает пример определенного выше глобального соотношения при $\alpha=-1$ для ряда

$$
\sum_{n=1}^{\infty}(-1)^{n} n \cdot n ! z^{n}
$$

3. Метод Зигеля-Шидловского и $F$-ряды. Общие теоремы. Для исследования арифметических свойств значений степенных рядов применяется метод Зигеля-Шидловского в теории трансцендентных чисел (см. [15]). Этот метод применим к широким классам рядов, так называемым $E$ и $G$-функциям Зигеля. При этом $E$-функции являются целыми функциями, а $G$-функции имеют конечный радиус сходимости в поле комплексных чисел.

Естественным шагом стало исследование рядов, имеющих нулевой радиус сходимости в поле комплексных чисел, но имеющих радиусы сходимости, большие 1 в любом поле $p$-адических чисел. Пусть

$$
f(z)=\sum_{n=0}^{\infty} c_{n} \cdot n ! \cdot z^{n} .
$$

Будем говорить, что этот ряд принадлежит классу $F\left(\mathbb{K}, C_{1}, C_{2}, C_{3}, q\right)$, если:

(1) все коэффициенты $c_{n}$ принадлежат некоторому алгебраическому числовому полю $\mathbb{K}$ конечной степени $\varkappa$ над полем $\mathbb{Q}$ рациональных чисел; 
(2) максимумы абсолютных величин алгебраически сопряженных с числом $c_{n}$ чисел представляют собой $O\left(e^{C_{1} n}\right), n \rightarrow \infty$ с некоторой постоянной $C_{1}$;

(3) существует такая последовательность натуральных чисел $d_{n}$, что при $k=0,1, \ldots, n$ числа $d_{n} c_{k}$ принадлежат кольцу целых чисел $\mathbb{Z}_{\mathbb{K}}$ поля $\mathbb{K}$ и $d_{n}=q^{n} d_{0, n}, q \in \mathbb{N}$, а числа $d_{0, n}$ делятся только на простые числа $p$, не превосходящие $C_{2} n$, и для всех таких простых $p$ выполняется неравенство

$$
\vartheta_{p}\left(d_{0, n}\right) \leqslant C_{3}\left(\log _{p} n+\frac{n}{p^{2}}\right) .
$$

Из свойства 3 следует, что если простое число $p$ не делит число $q$, то ряд (4), например, с рациональными коэффициентами $c_{n}$, имеет в поле $\mathbb{Q}_{p}$ радиус сходимости, равный $p^{1 /(p-1)}>1$. Известным примером $F$-ряда является ряд Эйлера

$$
\sum_{n=0}^{\infty} n ! \cdot(-z)^{n}
$$

Он представляет собой асимптотическое разложение для интеграла

$$
\int_{0}^{+\infty} \frac{e^{-w / z}}{1+w} d w
$$

Разумеется, если ряд (4) отличен от многочлена, он имеет в поле $\mathbb{C}$ нулевой радиус сходимости. Легко заметить также, что если коэффициенты $c_{n}$ ряда (4) удовлетворяют перечисленным условиям, то ряд

$$
\sum_{n=0}^{\infty} c_{n} \cdot z^{n}
$$

с теми же коэффициентами $c_{n}$ является $G$-функцией, а ряд

$$
\sum_{n=0}^{\infty} \frac{c_{n}}{n !} \cdot z^{n}
$$

с теми же коэффициентами $c_{n}$ является $E$-функцией в узком смысле.

Сформулированные ниже теоремы представляют собой некоторые аналоги основных теорем А. Б. Шидловского, справедливые для $F$-рядов.

Теорема 1 (см. [17, теорема 1.1]). Пусть $F$-ряды $f_{1}(z) \equiv 1, f_{2}(z), \ldots, f_{m}(z)$ линейно независимы над полем $\mathbb{K}(z)$ и составляют решение системы линейных дифференциальных уравнений

$$
y_{i}^{\prime}=Q_{i, 0}(z)+\sum_{j=1}^{m} Q_{i, j}(z) y_{j}, \quad i=1, \ldots, m,
$$

с коэффициентами из поля $\mathbb{C}(z)$ рачиональных функций от $z$. Пусть $\xi \neq 0, \xi \in \mathbb{K}$ - регулярная точка системь (5). Пусть

$$
L\left(y_{1}, \ldots, y_{m}\right)=h_{1} y_{1}+\ldots+h_{m} y_{m}
$$

- ненулевая линейная форма, $h_{i} \in \mathbb{Z}_{\mathbb{K}}, i=1, \ldots, m$. Пусть $H(L)=\max H\left(h_{i}\right)$. Найдется такая эффективно вычисляемая постоянная $H_{0}$, что для любого $H \geqslant \max \left(H_{0}, H(L)\right)$ существуют такие эффективно вычисляемая постоянная $C_{0}$, простое число $p$, удовлетворяющие неравенствам

$$
P_{l}(H)=l\left(\frac{\ln H}{\ln \ln H}\right) \leqslant p \leqslant u\left(\frac{\ln H}{\ln \ln H}\left(1+\frac{C_{0}}{\sqrt{\ln \ln H}}\right)\right)=P_{u}(H)
$$

и нормирование $v$ поля $\mathbb{K}$, продолэсающее р-адическое нормирование поля $\mathbb{Q}$, что имеет место неравенство

$$
L(\xi)=L\left(f_{1}(\xi), \ldots, f_{m}(\xi)\right) \neq 0 .
$$

Замечание. Можно также для хотя бы одного $v \in V_{1}$ установить оценку

$$
|L(\xi)|_{v} \geqslant H^{-m-\frac{m+3+2 m C_{5}}{\sqrt{\ln \ln H}}} .
$$


Теорема 2. Пусть F-ряды $f_{1}(z) \equiv 1, f_{2}(z), \ldots, f_{m}(z)$ составляют решение системы (5) и линейно независимы над полем $\mathbb{K}(z)$. Пусть $\xi \in \mathbb{K}$ - регулярная точка системы (5). Пусть

$$
L\left(y_{1}, \ldots, y_{m}\right)=h_{1} y_{1}+\ldots+h_{m} y_{m}-
$$

ненулевая линейная форма, $h_{i} \in \mathbb{Z}_{\mathbb{K}}, i=1, \ldots, m$. Тогда существует бесконечное множество таких простых чисел $p$ и нормирований $v$ поля $\mathbb{K}$, продолжающих р-адическое нормирование поля $\mathbb{Q}$, что в поле $\mathbb{K}_{v}$

$$
L\left(f_{1}(\xi), \ldots, f_{m}(\xi)\right) \neq 0 .
$$

Иными словами, ряды $f_{1}(\xi), \ldots, f_{m}(\xi)$ бесконечно линейно независимы.

Теорема 3. Пусть $F-р я д ы ~ f_{1}(z), f_{2}(z), \ldots, f_{m}(z)$ составляют решение системы (5) и алгебраччески независимы над полем $\mathbb{K}(z)$. Пусть $\xi \in \mathbb{K}$-регулярная точка системь (5). Пусть $P\left(y_{1}, \ldots, y_{m}\right)$ - ненулевой многочлен с коэфбициентами из $\mathbb{Z}_{\mathbb{K}}$. Тогда существует бесконечное множество таких простых чисел р и нормирований $v$ поля $\mathbb{K}$, продолжающих р-адическое нормирование поля $\mathbb{Q}$, что в поле $\mathbb{K}_{v}$

$$
P\left(f_{1}(\xi), \ldots, f_{m}(\xi)\right) \neq 0 .
$$

Иными словами, ряды $f_{1}(\xi), \ldots, f_{m}(\xi)$ бесконечно алгебраччески независимы.

4. Приложения общих теорем. Сформулированные теоремы имеют приложения к обобщенным гипергеометрическим рядам. Символ Похгаммера определен равенствами

$$
(a)_{0}=1, \quad a_{n}=a(a+1) \ldots(a+n-1), \quad n \geqslant 1 .
$$

Для множества действительных чисел $S=\left\{\alpha_{1}, \ldots, \alpha_{r} ; \beta_{1}, \ldots, \beta_{s}\right\}$, относительно которых предполагаем, что числа $\beta_{1}, \ldots, \beta_{s}$ - нецелые неположительные, принято обозначать символом

$$
{ }_{r} F_{s}\left(\begin{array}{c}
\alpha_{1} \ldots \alpha_{r} \\
\beta_{1} \ldots \beta_{s}
\end{array} ; z\right)=\sum_{n=0}^{\infty} \frac{\left(\alpha_{1}\right)_{n} \ldots\left(\alpha_{r}\right)_{n}}{\left(\beta_{1}\right)_{n} \ldots\left(\beta_{s}\right)_{n} n ! z^{n}}
$$

так называемый обобщенный гипергеометрический ряд. Можно рассматривать несколько более общие ряды вида

$$
\sum_{n=0}^{\infty} \frac{\left(\alpha_{1}\right)_{n} \ldots\left(\alpha_{r}\right)_{n}}{\left(\beta_{1}\right)_{n} \ldots\left(\beta_{s}\right)_{n}} z^{n} .
$$

Ряды (6) и (7) относятся к так называемым рядам Жевре порядков $s+1-r$ и $s-r$ соответственно.

Если $r-s<0$, то рассматриваемый ряд (7) представляет собой целую функцию (ряд (6) представляет целую функцию при $r-s \leqslant 0)$. $\mathrm{K}$ этим случаям относятся показательная функция, функции Бесселя, функции Куммера и большое количество других, важных в математике функций. При условии рациональности чисел $\alpha_{1}, \ldots, \alpha_{r} ; \beta_{1}, \ldots, \beta_{s}$ они входят в класс $E$-функций Зигеля, и к исследованию их значений применим известный метод Зигеля-Шидловского в теории трансцендентных чисел (см. [5]). Применению этого метода к гипергеометрическим $E$ функциям посвящены работы В. Х. Салихова [6-8], в которых получено близкое к полному решение проблемы. При $r-s=0$ ряд (4) имеет конечный радиус сходимости (ряд (3) имеет конечный радиус сходимости при $r-s=-1)$. K таким функциям относятся логарифмическая функция, гипергеометрическая функция Гаусса, многие алгебраические функции, неполные эллиптические интегралы и др. При условии рациональности чисел $\alpha_{1}, \ldots, \alpha_{r} ; \beta_{1}, \ldots, \beta_{s}$ они входят в класс $G$ функций Зигеля, и исследованию их посвящены работы А. И. Галочкина [1], Г. В. Чудновского [8], Э. Бомбьери [18], И. Андре [16] и др.

Если же $r-s>0$, то ряд (7), отличный от многочлена, имеет нулевой радиус сходимости в поле $\mathbb{C}$. (Ряд $(6)$ имеет нулевой радиус сходимости при $r-s \geqslant 0)$. В этом случае получаются $F$ ряды. Исследованию свойств таких рядов посвящены, например, работы $[2,10,12,13]$. В случае, когда среди параметров рассматриваемых гипергеометрических рядов содержатся иррациональные алгебраические числа, используя аппроксимации Эрмита-Паде, приведенные в [3], удается доказать бесконечную линейную независимость значений таких рядов в алгебраических точках (см. [11]). 


\section{СПИСОК ЛИТЕРАТУРЫ}

1. Галочкин А. И. Оценки снизу многочленов от значений аналитических функций одного класса// Мат. сб. - 1974. - 95 (137), № 3 (11). - С. 396-417.

2. Матвеев В. Ю. Алгебраическая независимость некоторых почти полиадических рядов// Чебышев. сб.. - 17, № 3. - С. 156-167.

3. Нестеренко Ю. В. Приближения Эрмита-Паде обобщенных гипергеометрических функций// Мат. сб. - 1994. -185 , № 3. - С. 39-72.

4. Новоселов E. В. Об интегрировании на одном бикомпактном кольце и его приложениях к теории чисел// Изв. вузов. Сер. мат. - 1961. - 3 (22). - С. 66-79.

5. Постников А. Г. Введение в аналитическую теорию чисел. - М.: Наука, 1971.

6. Салихов B. Х. Об алгебраической независимости значений гипергеометрических $E$-функций// Докл. AH CCCP. - 1989. - 307, № 2. - C. 284-286.

7. Салихов B. X. Неприводимость гипергеометрических уравнений и алгебраическая независимость значений E-функций// Acta Arithm. - 1990. - 53. - С. 453-471.

8. Салихов В. Х. Критерий алгебраической независимости значений одного класса гипергеометрических E-функций// Мат. сб. - 1990. - 181, № 2. - С. 189-211.

9. Фомин А. А. Числовые кольца и модули над ними. - М.: Прометей, 2013.

10. Чирский В. Г. Арифметические свойства полиадических рядов с периодическими коэффициентами// Докл. РАН. - 2014. - 459, № 6. - С. 677-679.

11. Чирский В. Г. Об арифметических свойствах обобщенных гипергеометрических рядов с иррациональными параметрами// Изв. РАН. Сер. мат. - 2014. - 78, № 6. - С. 193-210.

12. Чирский В. Г. Арифметические свойства полиадических рядов с периодическими коэффициентами// Изв. РАН. Сер. мат. - 2017. - 81, № 2. - С. 215-232.

13. Чирский В. Г. Арифметические свойства обобщенных гипергеометрических $F$-рядов// Докл. РАН. 2018. - 483, № 3. - C. 252-254.

14. Чирский В. Г., Матвеев В. Ю. О представлении натуральных чисел// Чебышев. сб. - 2013. - 14, № 1. - C. $75-86$.

15. Шидловский А. Б. Трансцендентные числа. - М.: Наука, 1987.

16. Andre Y. G-functions and geometry// in: Asp. Math. - Wiesbaden: Vieweg, 1989. - Vol. 13.

17. Bertrand D., Chiskii V., Yebbou J. Effective estimates for global relations on Euler-type series// Ann. Fac. Sci. Toulouse. - 2004. - 13, № 2. - P. 241-260.

18. Bombieri E. On G-functions// in: Recent Progress in Analytic Number Theory. — London: Academic Press, 1981. - P. 1-68.

19. Chudnovsky G. V. On applications of Diophantine approximations// Proc. Natl. Acad. Sci. U.S.A. - 1985. - 81. - P. 7261-7265.

Чирский Владимир Григорьевич

Московский государственный университет им. М. В. Ломоносова,

Московский педагогический государственный университет

E-mail: vgchirskii@yandex.ru 